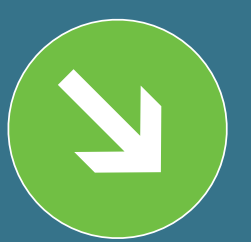

Ключевые слова:

станок, тепло-

вая деформация,

тепловая погреш-

ность, испытания,

тепловые смеще-

ния осей вращения,

температурные

измерения

\section{МЕТОДЫ ИСПЫТАНИЯ И УПРАВЛЕНИЯ ТЕПЛОВОЙ ЖЕСТКОСТЬЮ СТАНКОВ}

\author{
Алексей дОРОЖко
}

\begin{abstract}
Рассмотрены основные методики и процедуры испытаний для определения тепловых воздействий, вызванных различными тепловыми потоками, приводящими к искажению конструкции станка или системы позиционирования. Описаны методы измерения температур конструкции станка и базовые подходы к управлению и воздействию на тепловое состояние металлорежущих станков.
\end{abstract}

МЕТОДИКИ ИЗМЕРЕНИЯ ТЕПЛОВЫХ ДЕФОРМАЦИЙ

На сегодняшний день существует множество методов измерения смещений (погрешностей позиционирования и ориентации) деталей станка. Далеко не все эти системы могут использоваться для измерения тепловых погрешностей. Это обусловлено жесткими требованиями к процессу измерения тепловых деформаций:

$\rightarrow$ должно производиться измерение всех значимых геометрических погрешностей;

$\rightarrow$ необходимо производить измерение рабочего объема;

$\rightarrow$ неточность измерения должна быть достаточно низкой;

$\rightarrow$ измерения должны производиться за достаточно малое время - это позволит отслеживать воздействие изменений температур на параметры геометрических погрешностей (при помощи измерительной системы).

Измерительная система подбирается в зависимости от типа источников тепловых погрешностей станка. Воздействие внешних источников (например, температуры воздуха в производственном помещении) обычно приводит к медленным изменениям температуры конструкции станка, однако при этом данное воздействие влияет на всю объемную точность обработки. Внутренние источники нагрева (теплота, образующаяся в подшипниках и направляющих) приводят к возникновению местных деформаций конструкции станка и, как следствие, смещений, что приводит к частичному изменению объемной точности обработки. Смещения, вызываемые внутренними источниками нагрева, труднее прогнозировать, при этом величина этих смещений изменяется быстрее, чем величина смещений, вызываемых внешними источниками нагрева.

За последние 20 лет Международная организация по стандартизации (ISO) выпустила несколько стандартов: ISO 230-3 (тепловые смещения в станках); ISO 10791-10 (тепловые смещения в обрабатывающих центрах); ISO 13041-8 (тепловые смещения в токарных станках). Часть ISO 230 определяет процедуры испытаний для определения тепловых воздействий, вызванных различными тепловыми потоками, приводящими к искажению конструкции станка или системы позиционирования. Общепризнанным фактом является то, что предельная термоупругая деформация станка тесно связана с условиями эксплуатации. Условия испытаний, описанные в этой части ISO 230, предназначены не для моделирования нормальных условий эксплуатации, а для облегчения оценки рабочих характеристик и определения влияния окружающей среды на рабочие характеристики машины.

Например, использование охлаждающих жидкостей может существенно повлиять на фактическое тепловое поведение машины. Следовательно, эти испытания следует рассматривать только как предварительные испытания для определения фактического термоупругого поведения станка, если такое определение становится необходимым для определения характеристик станка. Тесты предназначены для измерения относительных смещений между компонентом, который удержи- 


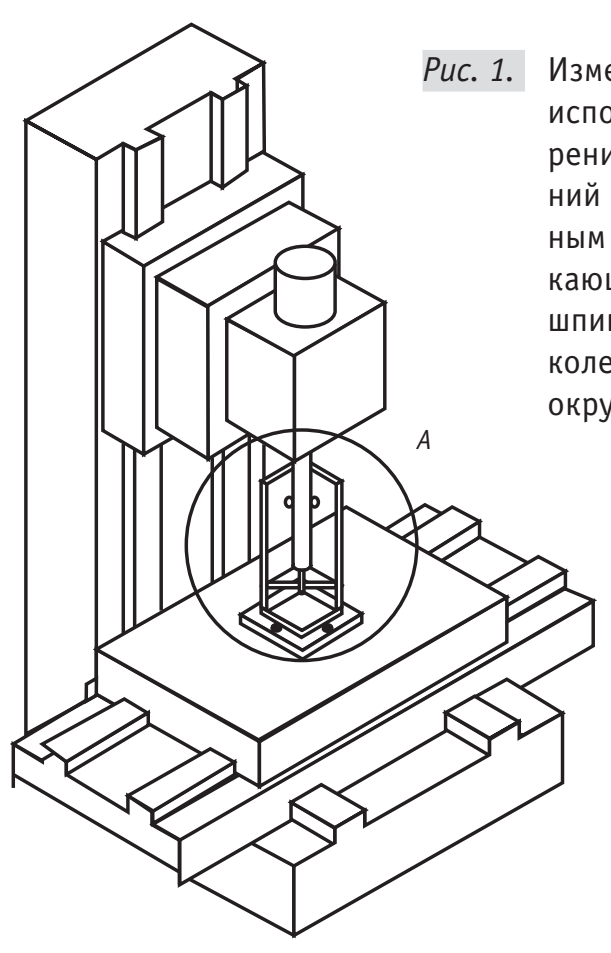

вает инструмент, и компонентом, который удерживает заготовку в результате теплового расширения или сжатия соответствующих элементов конструкции.

Испытания, указанные в этой части ISO 230, могут использоваться как для испытаний различных типов станков (типовые испытания), так и отдельных станков в целях приемки. Когда тесты требуются для приемочных целей, пользователь должен по согласованию с поставщиком / изготовителем выбрать те тесты, относящиеся к свойствам компонентов машины, которые представляют интерес. Простая ссылка на эту часть кода испытаний для приемочных испытаний без согласования применяемых частей и соответствующих сборов не может считаться обязательной для той или иной из договаривающихся сторон. Одной из существенных особенностей этой части ISO 230 является ее акцент на тепловых воздействиях окружающей среды на все эксплуатационные испытания, описанные в других частях ISO 230, связанные с измерениями линейного смещения (такими как точность линейного смещения, повторяемость и круговые испытания). Поставщик / производитель должен будет предоставить тепловые характеристики для среды, в которой машина может работать с указанной точностью. Пользователь машины будет нести ответственность за обеспечение подходящей тестовой среды, выполняя термические рекомендации поставщика / производителя или иным образом соглашаясь на снижение производительности.
В данных стандартах описываются методы систематического анализа тепловых характеристик станков, снабженных шпинделями. При помощи данной методики рассчитываются:

$\rightarrow$ погрешность отклонения температуры окружающей среды;

$\rightarrow$ тепловые смещения, возникающие при вращении шпинделя;

$\rightarrow$ тепловые смещения, возникающие при перемещении линейных осей.

В стандартах обычно описываются методики измерения тепловых смещений кромки инструмента и торца заготовки относительно друг друга. Обычно в процессе измерений заготовка закрепляется в шпинделе, на столе обрабатывающего центра (рис. 1) (или в инструментодержателе токарного станка) устанавливается измерительная установка, в конструкцию которой входят пять устройств измерения тепловых смещений.

Станок любой конструкции обладает тепловой резонансной частотой. Если станок подвергается тепловому нагружению в результате воздействия колебаний температуры внешней среды, любая частота может быть рассчитана при помощи метода переходной характеристики.

Для измерения тепловых смещений, возникающих при перемещении линейных осей, авторы стандартов рекомендуют регистрировать погрешности на обоих концах отрезка перемещения оси (рис. 2). При проведении подобных измерений тепловые смещения приводят к наклону оси, при этом угловые смещения на двух концах отрезка перемещения различаются. Если измерения 


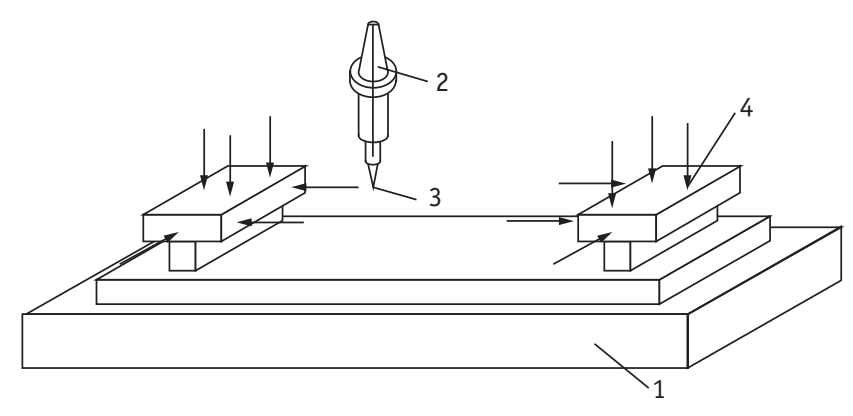

Puc. 2. Измерительная установка, снабженная датчиком касания, используемая для измерения тепловых смещений, возникающих при перемещении линейных осей обрабатывающего центра: 1 - стол станка, 2 - шпиндель станка, 3 - датчик касания, 4 - точки измерения (всего 12)

производятся только в одной точке, угловые смещения можно рассматривать как тепловые погрешности положения (отклонение от перпендикулярности для двух осей). При помощи лазерного интерферометра могут быть измерены осевые смещения вдоль отрезка перемещения оси в различных точках. Для регистрации тепловых смещений по двум направлениям в различных точках осей станка используются специальные сравнивающие устройства.

Для измерения воздействий изменения температуры окружающей среды на характеристики станков применяется специальная камера для проведения температурных испытаний. В камере осуществляется регулирование зависящей от времени температуры воздуха и температуры основания.

До настоящего времени в стандартах не даны описания способов измерения тепловых смещений осей вращения станков. При помощи устройства R-test и двух дополнительных наконечников для измерения длины осуществляется регистрация тепловых смещений по пяти направлениям, возникающих в процессе вращения шпинделя 5-координатного станка (измерения производятся для одного положения станка). При помощи калиброванного шарика и датчика R-test, устанавливаемого на столе станка, становится возможным измерение тепловых смещений по трем направлениям при различных положениях осей 5-координатного станка. Тепловые смещения вращающегося стола измеряются при помощи калиброванного шарика, установленного на столе. Увеличение температуры стола приводит к увеличению размеров стола. Данные измерения рекомендуется производить для оценки погрешностей заготовки, обрабатываемой на 5-координатном станке.

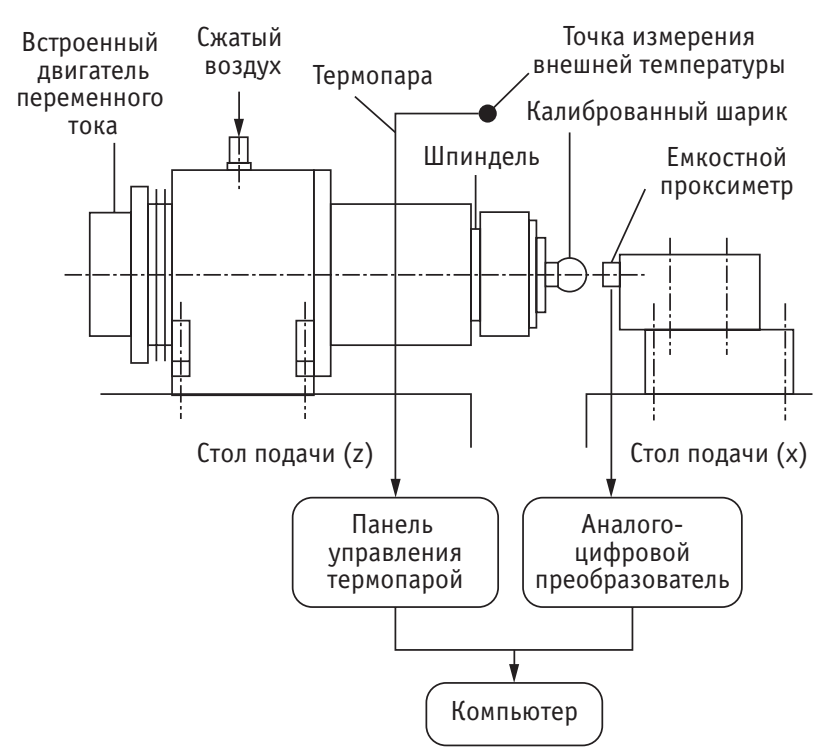

Puc. 3. Измерительная установка, используемая для измерения осевого расширения шпинделя (с применением емкостного измерительного датчика и калиброванного шарика)

Если для регистрации тепловых смещений центра инструмента при высоких скоростях перемещения (например, для регистрации тепловых смещений, возникающих в процессе вращения шпинделя) используются контактные датчики, в процессе измерений станок нужно выключать. По этой причине для измерения тепловых смещений центра инструмента, возникающих в процессе вращения шпинделя, гораздо чаще применяют бесконтактные, индуктивные и емкостные датчики (рис. 3).

Для регистрации смещений при помощи телескопического шарикового наконечника был разработан адаптер специальной конструкции, который устанавливается рядом со шпинделем в шпиндельной бабке, что позволяет производить измерения смещений центра инструмента (при необходимости регистрации воздействия характеристик шпинделя). При этом тепловые смещения самого адаптера приводят к погрешностям в измерениях. В горизонтальном направлении для минимизации воздействия адаптера была выбрана симметричная конструкция осей вращения (рис. 4).

В вертикальном направлении тепловые смещения адаптера минимизируются путем регулирования величины и направления теплового расширения. Данная регуляция осуществляется путем подбора различных материалов, различной геометрии конструкции адаптера, при помощи учета режимов теплового нагружения деталей адаптера. 


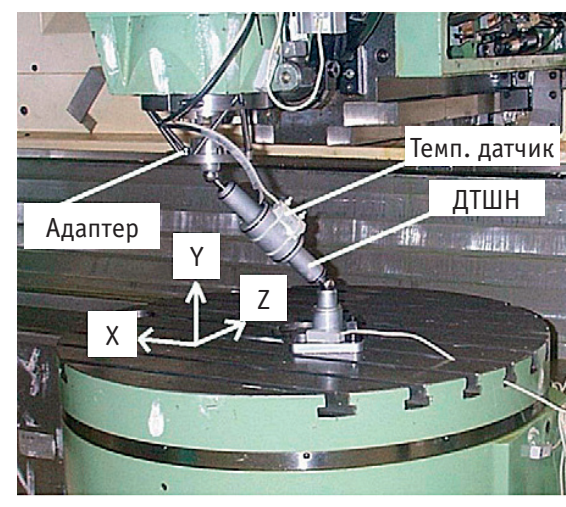

Puc. 4.

Измерительная установка, оснащенная двойным телескопическим шариковым наконечником (ДТШН) и предназначенная для измерения тепловых смещений 5-координатного станка

Значительными преимуществами обладают датчики касания, устанавливаемые непосредственно на шпинделе станка. При помощи одного такого датчика можно измерить тепловые смещения центра инструмента по трем направлениям, при этом также можно измерить тепловые смещения центральной оси шпинделя.

Датчики касания часто используются для измерения смещений в перерывах между операциями обработки. В подобных случаях вместо инструмента в инструментодержателе закрепляется датчик касания, используемый для регистрации тепловых смещений центра инструмента. Обычно на столе закрепляется измерительная установка, используемая для измерений в нескольких точках. Модель измерительной установки подбирается в зависимости от материала обрабатываемой детали, от материала станка, от типа материала с малым коэффициентом теплового расширения. Обычно для данных целей используются такие материалы как инвар, керамическое стекло; пластмасса, усиленная углеволокном, которая обладает очень малым коэффициентом теплового расширения по направлению волокон.

Процедура измерений, описываемая в данном стандарте, используется для станков, не подвергающихся нагружению и работающих в режиме чистовой обработки. С целью симуляции воздействия заготовки в некоторых случаях станок нагружается специальной массой. Гидравлический тормоз имитирует реактивный изгибающий момент от процесса резания, что позволяет произвести нагружение шпинделя (рис. 5).

Величина нагрузки зависит от давления масла и управляется при помощи гидравлического удара, регулируемого вручную. Нагруженный двойной шариковый наконечник (НДШН) используется для расчета статических характеристик станка, работающего в режиме нагружения.

Погрешности станка, вызываемые температурными изменениями во времени и пространстве, могут быть определены экспериментально при помощи исходных объектов с постоянной температурой или при помощи независимых измерений длин. Новые методики измерений основаны на многосторонности изменений, что позволяет осуществлять корректировку смещений деталей станка в режиме реального времени. К примеру, система M3D3, разработанная в Германии, использует несколько высокоточных следящих лазерных интерферометров для измерения положений в трехмерном режиме. Усложненные методики измерения позволяют осуществлять расчет погрешностей положения центра инструмента в режиме, практически свободном от возникновения погрешностей Аббе. Воздействия температуры внешней среды, давления воздуха, влажности рассчитываются при помощи лазерных измерительных систем, в работе которых используется формула Ciddor. Отдельные величины регистрируются при помощи нескольких датчиков, равномерно распределенных по всему объему измерений. В соответствии с подходом Kriging для расчета корректировки необходимо использовать карты температур, давлений, влажности.

Вне зависимости от возможности корректировки тепловых погрешностей очень важной является проблема расчета неточности измерений, что позволяет оценить качество процесса обработки. Обычно используются два метода. В основном в них для измерений применяются калиброванные элементы (шарик или диск с отверстиями), в другом - методики численного моделирования (к примеру, виртуальная координат-

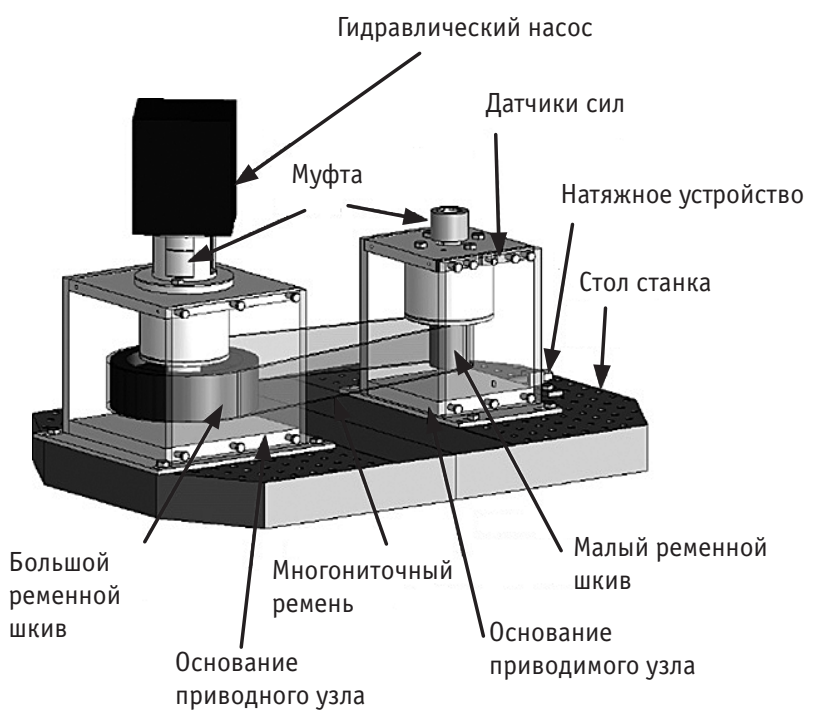

Puc. 5. Испытательная установка, используемая для измерения тепловых смещений в условиях нагружения (силы симулируются при помощи гидравлического насоса) 
но-измерительная машина (ВКИМ)). Второй метод обычно используется на станках с декартовыми координатными системами.

\section{ТЕМПЕРАТУРНЫЕ ИЗМЕРЕНИЯ}

Температурные измерения очень важны для повышения точности анализа тепловых погрешностей станков. Обычно для измерения температур конструкции станка используются термометры сопротивления. В прошлом для измерений широко использовались термометры сопротивления на основе платины Pt100 и Pt1000 (ввиду их практически линейных характеристик). При применении методик компьютерной линеаризации можно использовать другие типы термометров: термопары, терморезисторы с отрицательным температурным коэффициентом, терморезисторы с положительным температурным коэффициентом.

Из-за электромагнитной чувствительности термометров сопротивления при возникновении электрических полей появляются погрешности измерения. За последнее время были разработаны полупроводниковые термоэлементы (т.н. Smart Probes - «умные датчики»), обеспечивающие высокую электромагнитную стабильность.

Дискретные температуры конструкции станка измеряются при помощи контактных датчиков. В начале 60 -х годов для регистрации температурного распределения шпиндельной бабки токарного станка использовалась специальная флюоресцентная краска. Специальное химическое вещество поглощает свет при облучении источником излучения с частотой, отличной от частоты источника света. Величина поглощения света зависит от температуры. При исполь-

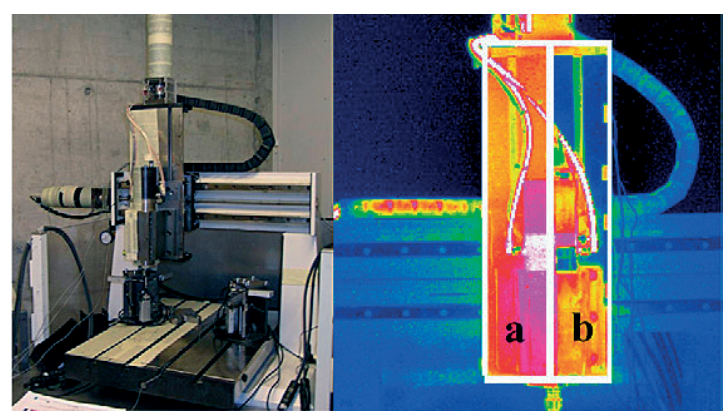

Puc. 6. Левая часть - 3-координатный фрезерный станок; правая часть - результаты инфракрасного измерения температур оси Z: a - поверхность, покрытая липкой лентой, $\mathrm{b}$ - неподготовленная металлическая поверхность корпуса шпинделя зовании данного метода погрешность измерения составила $\pm 1,65 \mathrm{~K}$.

В настоящее время для измерения температурных распределений поверхностей деталей станка обычно используются передающие тепловизионные камеры. Любой объект с температурой выше 0 К излучает электромагнитные волны. При увеличении температуры диапазон испускаемых электромагнитных волн смещается в зону коротких волн. Передающая тепловизионная камера регистрирует интенсивность инфракрасного излучения в зоне измерения. На основе этой информации программное обеспечение рассчитывает температуры. При использовании пирометрических измерительных систем можно регистрировать различные источники погрешностей. Излучение, регистрируемое измерительной системой, может быть испускаемым, отраженным и проходящим. Металлы обычно не пропускают инфракрасные волны. Коэффициенты отражения различных поверхностей станка значительно различаются. На рис. 6 показана инфракрасная схема станка. Сторона (а) вертикальной оси покрыта специальной клейкой лентой, рядом с ней расположен объект с определенным коэффициентом излучения. Сторона (b) является металлической поверхностью, отражающей излучение расположенных рядом элементов системы охлаждения.

Величина коэффициента отражения зависит от нескольких факторов: свойств материала, качества обработки поверхности, угла наклона камеры, величины шероховатости. На рис. 7 проиллюстрировано отражение теплоты на поверхностях образца Rugotest, подвергнутых токарной обработке (Ra 0,4-50 мкм). Различные области данной поверхности обладают различными коэффициентами отражения. Поверхности с малой шероховатостью Ra поглощают более инфракрасный свет. Вследствие этого уменьша-

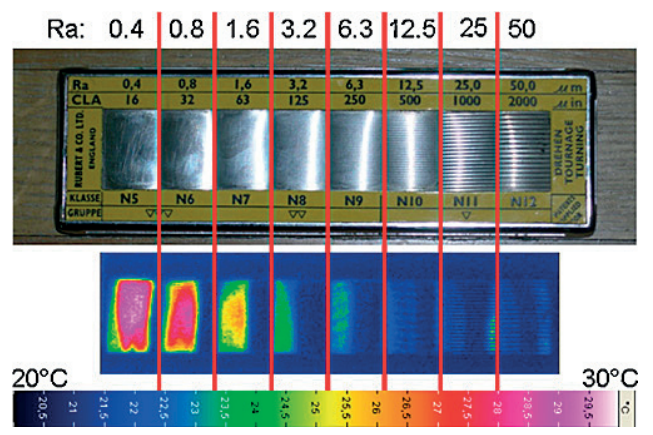

Puc. 7. Отражение теплоты на поверхностях образца Rugotest (после проведения токарной обработки шероховатость Ra составила 0,4-50 мкм, температура помещения $-20,5^{\circ} \mathrm{C}$ ) 


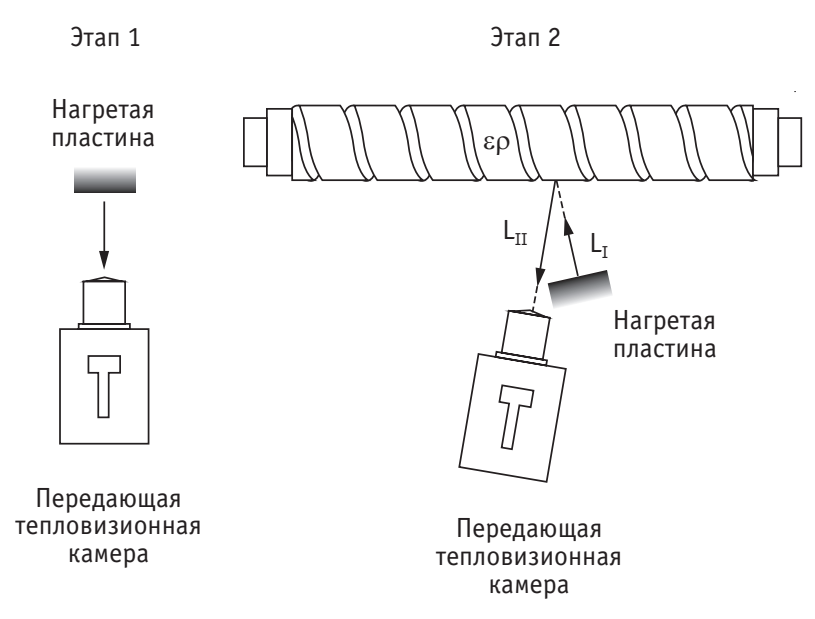

Puc. 8. Система двухэтапного измерения коэффициента излучения шпинделя шариковой винтовой пары. LI - излучение, испускаемое пластиной; LII - излучение LI, отражаемое шпинделем шариковой винтовой пары

ется коэффициент излучения данной поверхности.

Для измерения температуры шариковой винтовой пары использовали передающую тепловизионную камеру. Коэффициент излучения шариковой винтовой пары определяется за два этапа. На первом этапе измеряется излучение, испускаемое нагретой пластиной. На втором этапе измеряется излучение, отраженное шариковой винтовой парой. На основе разности этих двух величин рассчитывается коэффициент излучения шариковой винтовой пары. У всех поверхностей пары, кроме резьбовых, этот коэффициент составляет 0,169. В среднем резьбовые поверхности имеют коэффициент излучения, равный 0,34 . На рис. 8 проиллюстрированы два указанных выше этапа.

Для осуществления данной методики нагрев пластины должен быть равномерным. Если это условие не достигается, разность величин излучения приводит к неравномерности температур или к отличиям коэффициентов излучения в различных областях поверхности. Лучше всего для подобных измерений подходят небольшие пластины (для них легче добиться температурного выравнивания). По этой причине данная методика используется для расчета характеристик только деталей станка, но не характеристик всего станка.

На рис. 9 показан тепловой образ температурного распределения шариковой винтовой пары. Красные стрелки вдоль шариковой винтовой пары - это точки, в которых рассчитывается тепловое удлинение шариковой винтовой пары.
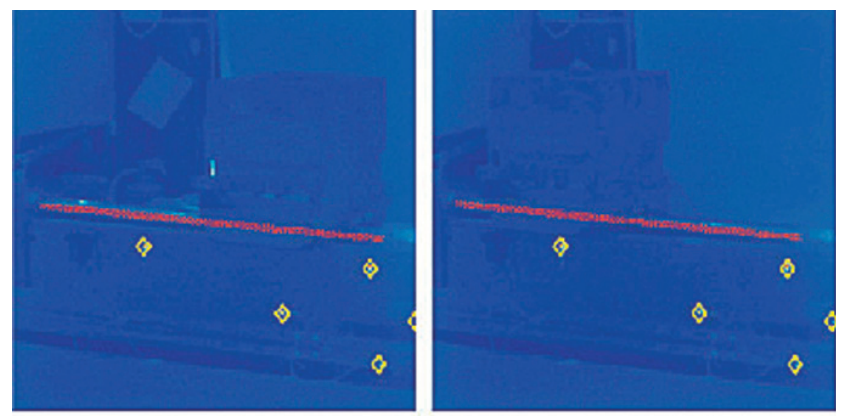

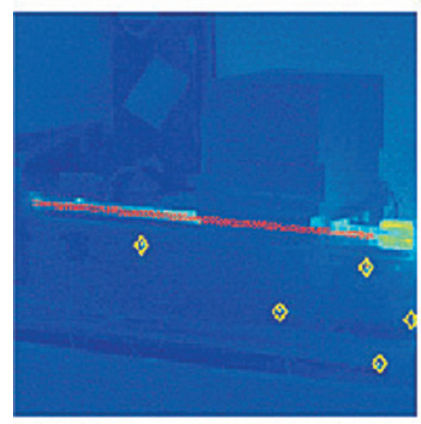

Смещения в начальной точке

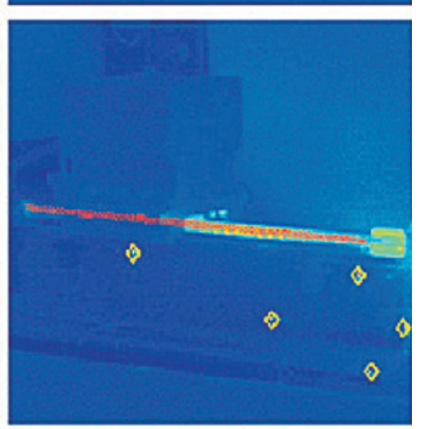

Смещения в конечной точке
Puc. 9. Термографический образ шариковой винтовой пары в холодном состоянии (верхние рисунки) и после 4 тыс. циклов обработки (нижние рисунки). Красные стрелки вдоль шариковой винтовой пары - это точки измерения температуры шариковой винтовой пары, желтые ромбы - это маркировки (контрольные точки)

Общей особенностью разработанных в России измерительно-диагностических комплексов и систем применительно к оценке тепловых характеристик станка является необходимость проведения полного натурного эксперимента большой длительности. При этом длительность тепловых испытаний станков до стабильного теплового режима может составлять 6-8 и более часов - в зависимости от тепловой инерционности станка. В отечественных источниках есть указание на то, что в последние годы занимаются вопросом сокращения тепловых испытаний.

Под сокращенными тепловыми испытаниями станков понимаются тепловые испытания, длительность которых во времени недостаточна для фиксации температурной стабилизации. Для сокращенных тепловых испытаний станков, исходя из анализа кинетики теплового процесса, наиболее актуальными являются три задачи:

$\rightarrow$ выбор функции экстраполяции;

$\rightarrow$ выбор минимального времени проведения тепловых испытаний;

$\rightarrow$ оценка достоверности полученных оценок. 


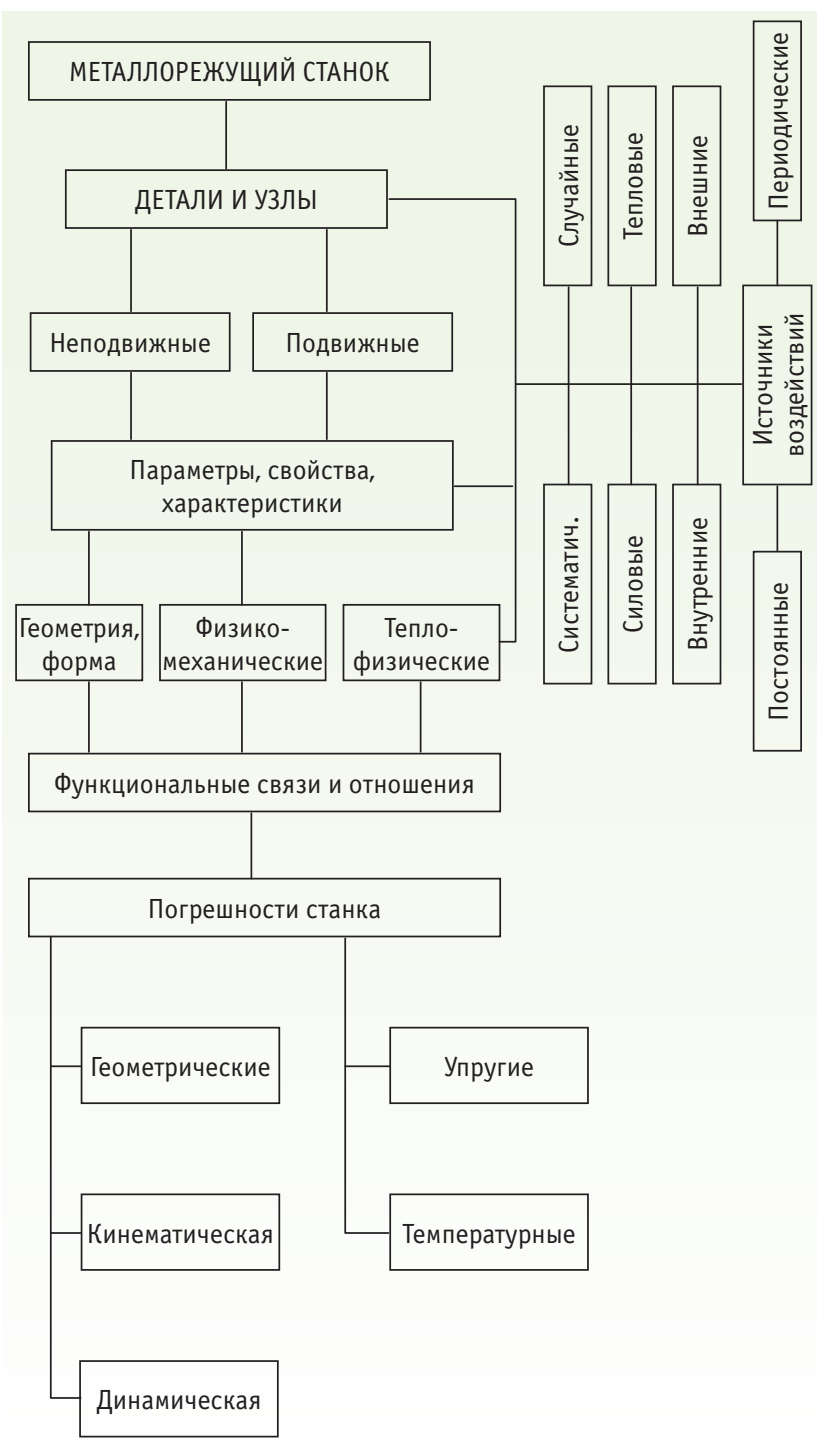

Puc. 10. Погрешности станка при силовых и тепловых воздействиях

Анализ результатов машинных испытаний показал, что при выборе погрешности прогнозирования теплового состояния станка на уровне $10 \%$ от установившихся температур сокращение длительности тепловых испытаний обеспечивается не менее, чем на $50 \%$.

Был разработан формализованный метод оценки тепловых характеристик металлорежущих станков, обеспечивающий повышение точности прогнозирования и сокращение трудоемкости и сроков натурных испытаний. Экспериментальная апробация разработанных алгоритмов показала, что погрешность оценки для тепловой постоянной времени первой моды не превышает $10 \%$, что обеспечивает погрешность прогнозирования температур и темпера- турных перемещений не более 5\%. Однако сами авторы признают главный недостаток этого метода, который заключается в том, что оценка времени температурной стабилизации станка по температурным перемещениям и температурам в различных точках станка, измеренным на деталях его несущей системы, не совпадают. В общем случае, выход на установившийся режим станка по температурным перемещениям фиксируется раньше. Несовпадение оценок для параметра тепловой характеристики может приводить к тому, что после фиксации максимальных температурных перемещений происходит уменьшение температурных перемещений. Это объясняется перераспределением тепловых потоков по отдельным деталям станка. Этот факт накладывает ограничения на область использования разработанного метода, который не может учесть непрогнозируемый характер изменения тепловых деформаций станка, проявляемый только при большой длительности натурных испытаний.

Указанные на рис.10 погрешности являются измеряемыми величинами, которые дают объективную оценку свойств станка, в том числе и способности станка к сопротивлению к силовым и тепловым воздействиям.

Очевидно, что наиболее желательной характеристикой являются показатели, которые однозначно определяют эту способность станка. Так, известно понятие жесткость как способность сопротивляться силовым воздействиям, которая имеет размерность [н(кгс)/мкм], а способ контроля определяется ГОСТ 7035-75. Для тепловых воздействий имеются попытки аналогичным образом определить понятие «термической жесткости», как способность конструкции препятствовать термическим воздействиям и определять как частное от деления потерь мощности на возникшую максимальную деформацию в выбранных координатах при определенном числе оборотов.

Количественное значение этого параметра определяется как отношение потерь мощности, возникающих при проведении испытаний к максимальному изменению выходного параметра станка по координатам за это же время. Также предлагается параметр свойства конструкции противодействовать термическим возмущениям. В количественном выражении для линейных величин это мкм/кВт·ч, а для угловых град/кВт·ч по каждому выходному параметру станка, как отношение величины перемещения к моменту окончания очередного цикла термического воздействия к величине потребленной энергии за это время. 
Из этого следует очевидная сущностная разница применения понятий жесткость и термическая жесткость, так как в первом случае четко фиксировано место приложения силы и место измерений, а во втором случае фиксировано то же самое место измерения, а величина и место теплового воздействия являются неопределенными величинами. Очевидно, что такая «объективная» оценка не может служить основанием для управления тепловым поведением станка.

Важной темой современных исследований является расчет смещений центра инструмента на основе измеренного температурного распределения станка. Для осуществления компенсации смещений центра инструмента на основе данных по температурному распределению должны быть выведены различные постоянные времени и решены дополнительные задачи. В процессе эксплуатации станка легче измерить местные температуры, чем смещения центра инструмента. Алгоритмы компенсации для станка подбираются в зависимости от выбранных точек измерения температуры.

Обзору, обобщению и анализу реализации методов управления и воздействия на тепловое состояние металлорежущих станков посвящены обобщающие работы, в которых дана классификация рассматриваемых методов:

$\rightarrow$ уменьшение энергетического потенциала источников нагрева;

$\rightarrow$ управление тепловым потоком;

$\rightarrow$ проектирование теплоустойчивой конструкции станка;

$\rightarrow$ компенсация тепловых деформаций;

$\rightarrow$ управления процессом коррекции погрешностей, которые зависят исключительно от температуры, или зависят и от положения, и от температуры.

Отмечается также, что существующие методы компенсации основаны на:

$\rightarrow$ непосредственных, прямых методах измерения тепловых погрешностей;

$\rightarrow$ косвенных методах: по измеренной температуре в точках станка и расчету по тепловой модели;

$\rightarrow$ косвенных методах по измеренному параметру (например, частоте вращения, скорости перемещения по осям и т.п.) и расчету по построенной или выбранной математической модели;

$\rightarrow$ комбинированные методы из вышеперечисленных;

$\rightarrow$ других методах управления, в том числе и комбинации предыдущих.

В качестве математических методов построения моделей, которые связывают взаимодействующие параметры, применяются методы:

$\rightarrow$ линейной и нелинейной регрессии; $\rightarrow$ нейронные сети;

$\rightarrow$ нечеткой логики;

$\rightarrow$ передаточной функции;

$\rightarrow$ теории Грей-систем;

$\rightarrow$ В-сплайны;

$\rightarrow$ матрицы однородных преобразований.

Функциональная связь между измеренной температурой в узлах станка и величиной смещений можетбытьустановлена экспериментально. При хорошей повторяемости и достаточно стабильной воспроизводимости результата эта функциональная связь выразится уравнением, которое может быть положено в основу работы управляющего устройства для компенсации тепловых деформаций.

\section{ЛИТЕРАТУРА}

1. Кузнецов А. П. Методы оценки и контроля качества металлорежущих станков с ЧПУ. - М.: ВНИИТЭМР, 1985. 68 с.

2. Кузнецов А.П. Тепловое поведение и точность металлорежущих станков. - М.: Янус-К, 2011. 256 с.

3. Кузнецов А.П. Критерии подобия теплового поведения деталей и узлов металлорежущих станков. - М.: Вестник машиностроения. 2011. № 4. C. 57-63.

4. ISO 230-3 -2007, Test code for machine tools.Part 3: Determination of thermal effects.

5. Yto Y. Thermal deformation in Machine tools. McGraw-Hill, 2010. 214 p.

6. Mayr J., Jedrzejewski J., UhImann E., Donmez M. A., Knapp W., Hartig F., Wendt K., Moriwaki T., Shore P., Schmitt R., Brecher C., Wurz T., Wegener K. Thermal issues in machine tools // CIRP Annals-Manufacturing Technology. 2012. T. 61. PP. 771-791.

7. Дмитриев Б. М. Исследование точности станков методами термодинамики // Технология машиностроения. 2016. № 8. С. 46-50.

8. Дмитриев Б. М. Термическая жесткость несущей системы металлорежущего станка // Вестник машиностроения. 2017. № 11. С. 56-59.

9. Kuznetsov A.P., Koriath H.-J., Dorozhko A. O. The Methods for Controlled Thermal Deformations in Machine Tools.Conference on Thermal Issues in Machine Tools. Proceedings CIRP sponsored conference.Verlag Wissenschaftliche scripten. Dresden, 2018. PP. 47-60.

\section{ДОРОЖко Алексей Олегович - \\ магистр техники и технологии МГТУ «СТАНКИН», начальник отдела Департамента \\ станкостроения и инвестиционного \\ машиностроения Минпромторга России}

